\title{
Design, characterization and in vivo functioning of a light-dependent histidine protein kinase in the yeast Saccharomyces cerevisiae
}

\author{
Aleksandra Bury $^{*+}$ (D) and Klaas J. Hellingwerf ${ }^{\dagger}$
}

\begin{abstract}
Helical alignment of the a-helical linker of the LOV (light-oxygen-voltage) domain of YtvA from Bacillus subtilis with the a-helical linker of the histidine-protein kinase domain of the $\operatorname{SIn} 1$ kinase of the phospho-relay system for osmoregulation of Saccharomyces cerevisiae has been used to construct a light-modulatable histidine protein kinase. In vitro, illumination with blue light inhibits both the ATP-dependent phosphorylation of this hybrid kinase, as well as the phosphoryl transfer to Ypd1, the phosphoryl transfer domain of the SIn1 system. The helical alignment was carried out with conservation of the complete Ja helix of YtvA, as well as of the phosphorylatable histidine residue of the SIn1 kinase, with conservation of the hepta-helical motive of coiled-coil structures, recognizable in the helices of the two separate, constituent, proteins. Introduction of the gene encoding this hybrid histidine protein kinase into cells of S. cerevisiae in which the endogenous $\ln 1$ kinase had been deleted, allowed us to modulate gene expression in the yeast cells with (blue) light. This was first demonstrated via the light-induced alteration of the expression level of the mannosyltransferase $\mathrm{OCH} 1$, via a translational-fusion approach. As expected, illumination decreased the expression level of $\mathrm{OCH}$; the steady state decrease in saturating levels of blue light was about $40 \%$. To visualize the in vivo functionality of this light-dependent regulation system, we fused the green fluorescent protein (GFP) to another regulatory protein, HOG1, which is also responsive to the SIn1 kinase. HOG1 is phosphorylated by the MAP-kinase-kinase Pbs2, which in turn is under control of the SIn 1 kinase, via the phosphoryl transfer domain Ypd1. Fluorescence microscopy was used to show that illumination of cells that contained the combination of the hybrid kinase and the HOG1::GFP fusion protein, led to a persistent increase in the level of nuclear accumulation of HOG1, in contrast to salt stress, which - as expected — showed the well-characterized transient response. The system described in this study will be valuable in future studies on the role of cytoplasmic diffusion in signal transduction in eukaryotic cells.
\end{abstract}

Keywords: YtvA, Cytoplasmic diffusion, SIn1, Wall stress, Two-component regulation system, Nuclear shuttling

\section{Introduction}

During the last decade of the previous century, progress in the dynamic resolution of protein structure, in the availability of genomic DNA sequence information, and in the synthetic biology of the heterologous production

\footnotetext{
*Correspondence: a.bury@uva.nl

${ }^{\dagger}$ Aleksandra Bury and Klaas J. Hellingwerf contributed equally to this work Molecular Microbial Physiology Group, Swammerdam Institute for Life Sciences, University of Amsterdam, Science Park 904, 1098 $\mathrm{XH}$ Amsterdam, The Netherlands
}

of complex holo-proteins, have brought our understanding of the molecular basis of cellular signal transduction networks down to the atomic level (see e.g. (Ridge et al. 2003)). This development was aided by the modular nature of many signal transduction proteins, which is particularly notable in the dominant type of prokaryotic signal transduction network, the 'two-component regulatory system', including its more complex variant, the 'phosphorelay system' (Nixon et al. 1986; Burbulys et al. 1991). In this development photosensory receptor proteins did play an important role because of the ease 
and accuracy with which these proteins can be (de)activated (for review see e.g. (van der Horst and Hellingwerf 2004; Hoff et al. 1997)). Understanding of the atomic basis of the structural and dynamic aspects of the transitions between the receptor- and the signalling state of signal transduction proteins then led to the development of rational and intuitive guidelines to combine functional (input/output) domains into new functional chimera's, as could be concluded from analyses of their performance both in vitro and in vivo (Levskaya et al. 2005; Wu et al. 2009; Möglich et al. 2009).

These technical developments, and the derived improved insight, have led to the emergence of the interdisciplinary research field of 'optogenetics' (Miller 2006; Ernst et al. 2008; Zhang et al. 2010). This field meanwhile has made radically new and very important contributions to the disciplines of both cell biology (Bacchus and Fussenegger 2012) and neurobiology (Kim et al. 2017). Gradually, these developments now also start to impregnate the field of biotechnology, including the area of sustainability applications of 'direct conversion' (Savakis and Hellingwerf 2015) with cyanobacteria (Abe et al. 2014; Miyake et al. 2014).

Complete understanding of cellular signal transduction networks, however, not only requires understanding of the dynamics of the structural transitions within the protein components involved, but-particularly for those operating in the larger, i.e. mostly eukaryotic, cells-also resolution of the spatial dimension of such processes. This latter aspect is not only dictated by association/dissociation kinetics of the underlying physicochemical signals (e.g. an electric field or osmotic pressure), signaling molecules and signal-transmission- and output proteins, but also by the processes of classical- and/or anomalous diffusion of all these components, either in the cytoplasm or in the cytoplasmic membrane, with possibly additional effects of molecular crowding.

To resolve (part of) these latter aspects, it would be of great value to have a signal-transduction system available that can be triggered with (a flash of) visible light, and that initiates relocation of a specific component of that signal transduction network in the cell, like e.g. between subcellular compartments. Here we report the design, construction and in vitro and in vivo testing of such a network. Our approach is based on the construction of a chimeric histidine protein kinase, composed of the lightoxygen-voltage, LOV, domain of the stressosome protein YtvA from Bacillus subtilis (van der Steen et al. 2012) as the signal input domain and the histidine-protein kinase domain of the Sln1 kinase (Li et al. 2002) of a two-component regulatory system of the yeast Saccharomyces cerevisiae as the output domain, for relay of the (light) signal to the downstream components.
The Sln1 kinase of S. cerevisiae is part of the 'wall stress' signal transduction network of this yeast (for a brief overview: see Fig. 1) and has the typical structure of a phospho-relay system (Gao and Stock 2009; Fassler and West 2010). Its input kinase is located in the cytoplasmic membrane of yeast cells and able to convert signals derived from damage of components of their cell wall and of (a) signal(s) derived from osmotic stress, into changes in the level of phosphorylation of the cytoplasmic phosphoryl transfer domain, Ypd1 (Ferrigno et al. 1998). The level of phosphorylation of Ypd1 modulates nuclear gene expression directly (e.g. of Skn7), and also indirectly-via the MAP kinase pathway of the Ssk system-through the shuttling of the transcriptional regulator HOG1 between the cytoplasmic and nuclear compartment (Lu et al. 2003). Via analysis of the spatial distribution of fluorescent reporters in fixed Saccharomyces cells, sampled after triggering of either the natural- or an engineered LOV::Sln1-containing signal transduction network, we have been able to show the functionality of the designed chimeric light-dependent histidine protein kinase.

\section{Materials and methods Growth of S. cerevisiae}

The starting strain $\triangle \mathrm{YLR} 113 \mathrm{~W}$ (Additional file 1: Table S2) was cultivated on rich medium (YPD) agar plates, followed by growth in YPD liquid medium, at $30^{\circ} \mathrm{C}$ in a shaking incubator, followed by growth on minimal complete medium for gene knock out and plasmid transformation experiments. For selection of the $\operatorname{Sn} 1$ knockout strain, nourseothricin sulphate (clonNAT) was added to the minimal complete medium. To select for the continued presence of the plasmids, carrying the required customized version of the genes constructed, minimal drop out media were used.

\section{Molecular genetics and protein purification}

Genes encoding a required sequence (e.g. of a hybrid kinase) were amplified via PCR and cloned into a $\mathrm{PQE}$ vector (Qiagen, Hilden, Duitsland) for heterologous overexpression as a poly-histidine tagged protein in E. coli, and in a pRSII (Chee and Haase 2012) vector for in vivo expression in S. cerevisiae. DNA-fusion constructs were generated by overlap-extension PCR. After gene expression in E. coli, the recombinant proteins were purified from the cell-free extracts in a two-step procedure that makes use of: (i) Affinity chromatography on a HisTrap FF column (GE Healthcare, Chicago, Illinois, United States, $5 \mathrm{~mL}$ column) and (ii) Anion exchange chromatography on a ResQ column (GE Healthcare, Chicago, Illinois, United States $6 \mathrm{ml}$ column volume). For all proteins containing the LOV domain, their concentration was determined using the extinction coefficient of 


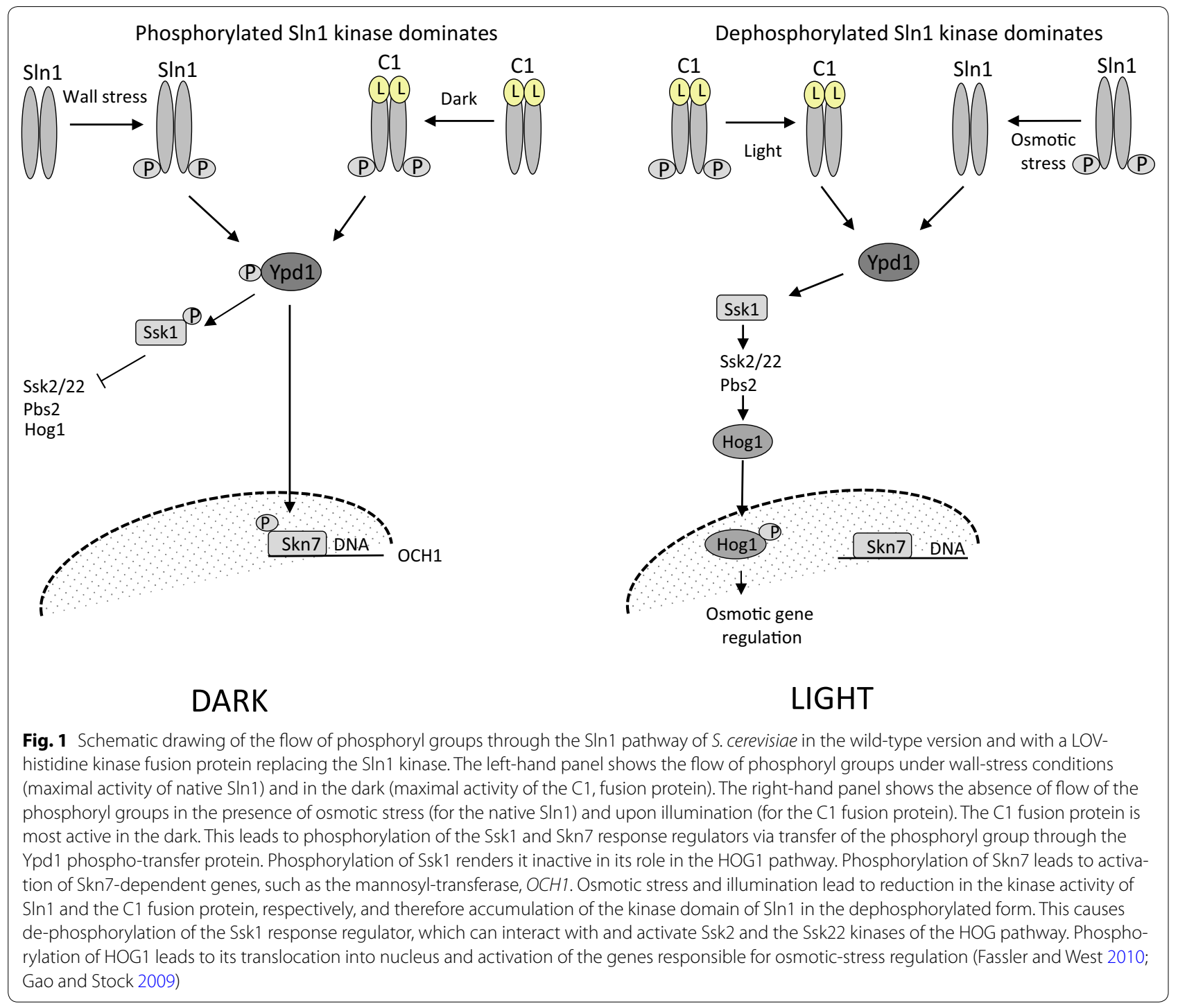

$14,000 \mathrm{M}^{-1} \mathrm{~cm}^{-1}$ at $450 \mathrm{~nm}$ (Koziol 1971). The Bradford method was used for all other proteins (Bradford 1976) (see also Additional file 1: Tables S1 and S2).

\section{In vitro assay of the extent and rate of phosphorylation} of the hybrid histidine protein kinases and of phosphoryl transfer from SIn 1 kinase- to the Ypd1 phosphoryl transfer domain

Kinase activity assays were carried out after slight modification of established procedures (Fassler and West 2010): $30 \mu \mathrm{M}$ of the specific histidine protein kinase was incubated with 1-5 $\mathrm{mM}$ cold ATP, after mixing of the latter with $3300 \mathrm{Ci} / \mathrm{mmol}$ of $\left[\gamma^{-}{ }^{32} \mathrm{P}\right]$-ATP. All reactions were carried out in a buffer containing $50 \mathrm{mM}$ Tris. $\mathrm{HCl}$ $\mathrm{pH}=8,100 \mathrm{mM} \mathrm{KCl}, 15 \mathrm{mM} \mathrm{MgCl}, 2 \mathrm{mM}$ DTT and 20\% $(\mathrm{v} / \mathrm{v})$ glycerol in a total volume of $0.1-1 \mathrm{ml}$ in Eppendorf tubes. Time-series samples were taken between 0 and $30 \mathrm{~min}$ at regular intervals. Samples were immediately mixed with $33 \%$ (v/v) fourfold concentrated stop buffer. This concentrated stop buffer contains $0.25 \mathrm{M}$ Tris. $\mathrm{HCl}$ $\mathrm{pH}=8,8 \%(\mathrm{w} / \mathrm{v})$ SDS, $40 \%(\mathrm{v} / \mathrm{v})$ glycerol, $40 \mathrm{mM}$ EDTA, $0.008 \%(\mathrm{w} / \mathrm{v})$ bromophenol blue and $4 \mathrm{mM} \beta$-mercaptoethanol. For the phosphoryl transfer experiments, the histidine kinases were first autophosphorylated for $1 \mathrm{~h}$ in the dark, after which Ypd1 was added to the sample in a molar ratio of 1:2. Time-series samples were taken between 1 and $30 \mathrm{~min}$. Samples were immediately mixed with stop buffer, just as described above. Samples were analyzed on $10 \%(\mathrm{w} / \mathrm{v})$ SDS PAGE gels, which were then exposed to a GE Healthcare screen (GE Healthcare, Chicago, Illinois, United States). Screens were scanned with a Typhoon Fla 7000 system (GE Healthcare, Chicago, 
Illinois, United States) and the resulting data files were saved as.tiff files. Image Quant software (GE Healthcare, Chicago, Illinois, United States) was used for the quantification of the intensity of the different bands. Kinase phosphorylation experiments and phosphoryl transfer experiments were conducted in the dark, with minimal red background light (Avila-Perez et al. 2006), or under constant illumination from blue light emitting diodes (LEDs with $\lambda^{\max }=464 \mathrm{~nm}$ ) with an incident light intensity of $200 \mu$ Einstein $\mathrm{m}^{2} \mathrm{~s}^{-1}$.

\section{Activation of the $\operatorname{SIn} 1$ kinase domain in vivo with (stress) signals}

For the application of the osmotic stress signal, $0.4 \mathrm{M}$ $\mathrm{NaCl}$ (final concentration) was added to $2 \mathrm{ml}$ cell suspension, growing exponentially in minimal selection medium, at $30{ }^{\circ} \mathrm{C}$, on a rotary shaker in $12 \mathrm{ml}$ glass tubes (Fassler and West 2010). Light activation of the hybrid LOV-kinase protein was achieved with blue LEDs with $\lambda^{\max }=464 \mathrm{~nm}$, with an incident light intensity of $200 \mu$ Einstein $\mathrm{m}^{2} \mathrm{~s}^{-1}$. Cells were fixed with $0.37 \%(\mathrm{v} / \mathrm{v})$ $p$-formaldehyde and rapidly frozen in liquid nitrogen for further analysis (Fassler and West 2010).

\section{Measurement of the level of expression of reporter enzyme via $\beta$-galactosidase activity}

Overnight cultures of recombinant strains of $S$. cerevisiae were grown in yeast extract peptone dextrose (YPD) medium in the dark, starting from a single colony from a plate of the minimal selection medium. The overnight cultures were diluted to $\mathrm{OD}_{600}=0.05$ and allowed to grow in the dark or in the light for $6 \mathrm{~h}$. Dark cultures had been wrapped tightly in tinfoil. Dark samples were taken with minimal red background light intensity (Avila-Perez et al. 2006; see above). Samples from illuminated cultures were taken under constant illumination with blue LEDs $\left(\lambda^{\max }=464 \mathrm{~nm}\right)$ with an incident intensity of $200 \mu$ Einstein $\mathrm{m}^{2} \mathrm{~s}^{-1}$. Samples were immediately transferred to an ice/water mixture and immediately flash frozen with liquid nitrogen for subsequent storage at $-80{ }^{\circ} \mathrm{C}$. $\beta$-galactosidase activity was measured in the cells from all samples and expressed in Miller units, based on the average value of at least 8 independently isolated transformants (Miller 1972).

\section{Microscopy}

Log-phase cultures of the yeast $S$. cerevisiae, expressing a HOG1::GFP (green fluorescent protein) fusion protein, were fixed with $0.37 \%(\mathrm{v} / \mathrm{v}) p$-formaldehyde for $1 \mathrm{~h}$, washed, re-suspended in phosphate-buffered saline (PBS) $\mathrm{pH}=7$, and stained with $0.5 \mu \mathrm{g} 4,6$-diamidino2-phenylindole (DAPI) per $\mathrm{ml}$ culture to visualize the nuclei of the cells. The yeast cells were observed using a Nikon Eclipse $\mathrm{Ti}$ inverted microscope (Shinagawa, Tokyo, Japan), equipped with a $100 \times$ objective. Fluorescence emission signals of GFP and DAPI were generated using a Lumencor (Beaverton, United States) fluorescent light source and detected at 470 and $395 \mathrm{~nm}$, respectively. Images were captured using a Hamamatsu digital camera C11440 (Hamamatsu City, Japan) driven by the Nikon elements AR 4.50.001 software (Shinagawa, Tokyo, Japan). All pictures of cells with a specific fluorophore were acquired using the same exposure time: $100 \mathrm{~ms}$ for DAPI and $400 \mathrm{~ms}$ for GFP. The pictures were then analyzed using ImageJ software (Schindelin et al. 2012) without further manipulation. For analysis, images were exported as.tiff files for import into ImageJ software. For quantitative analysis of the microscopy data, pictures of cells with DAPI-stained nuclei, and with HOG1::GFP expression, were overlapped. Cells with nuclear- and cytoplasmic localization (only cells with $>1.5$-fold nuclear accumulation were counted as positive; by definition the others as cells with cytoplasmic localization) of the HOG1::GFP reporter protein were counted and the percentage of cells with nuclear localization was calculated and plotted.

\section{Results}

\section{In vitro phosphorylation assays}

The helical linker regions of YtvA and Sln 1 were aligned according to the hepta-helical pattern of the coiled-coil structure that presumably is present in both of them, and joined in several different ways (Additional file 1: Table S1 and Fig. 2a), i.e. with preservation of the (J $\alpha-)$ helix from either protein completely, or partially; with or without insertion of extra amino acids to translationally shift the hepta-helical pattern and with or without conservation of the position of the crucial phosphorylatable histidine of the Sln1 kinase domain. The resulting hybrid kinases, with the truncated $\operatorname{Sin} 1$ kinase domain as a reference, were assayed for kinase activity in the dark with the classical kinase assay based on the use of ${ }^{32} \mathrm{P}[\mathrm{ATP}]$. Other assays, e.g. based on inorganic phosphate release, were tested too, but turned out to be less suited.

Of the initial series of hybrid kinases tested (i.e. $\mathrm{C} 1$ to $\mathrm{C} 8$ ), only the truncated reference domain and the $\mathrm{C} 1$, $\mathrm{C} 2$ and $\mathrm{C} 6$ fusion proteins showed considerable autophosphorylation activity, in the order of $0.38,0.24$ and $0.24 \mathrm{nmolP} / \mathrm{g}$ protein $/ \mathrm{min}$ for the latter three, respectively, at saturating concentrations of the nucleotide substrate (i.e. $5 \mathrm{mM}$; see (Fassler and West 2010)) and $30 \mu \mathrm{M}$ of the specific histidine protein kinase (domain). Next, we tested a possible difference between this activity, and the corresponding activity in saturating intensities of blue light (for further experimental detail: see Materials and methods). These assays revealed that significant 


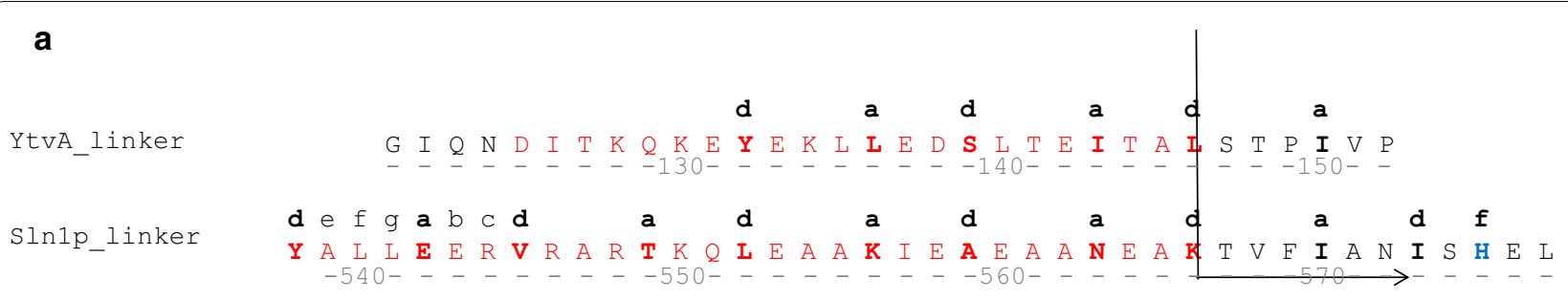

b

C1 autokinase activity

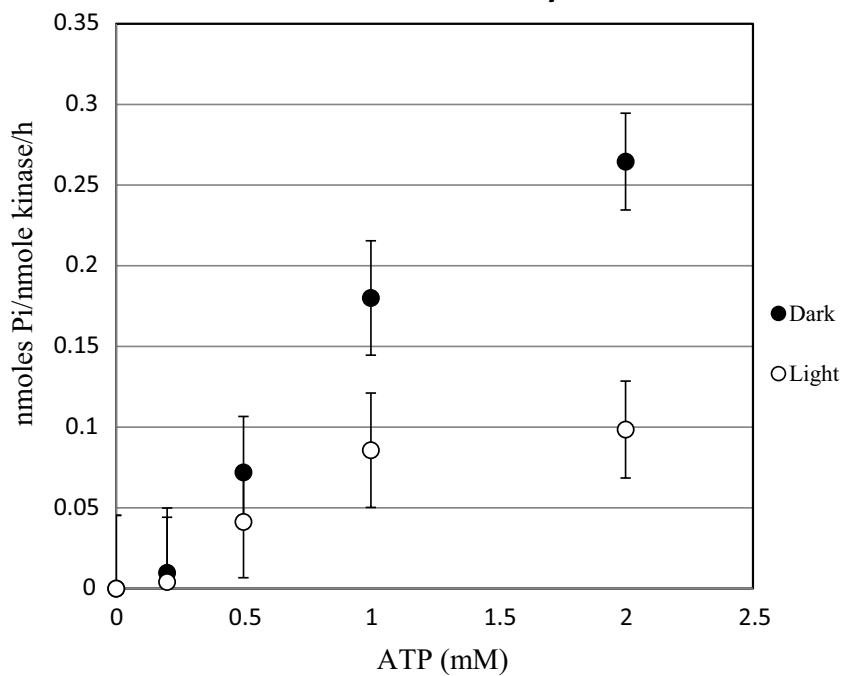

Fig. 2 a Alignment of the coiled coil structures from $\operatorname{SIn} 1$ and YtvA. The point of switching over from the amino acid sequence of the Ja helix of YtvA to the SIn 1 sequence in the $\mathrm{C} 1$ construct is indicated by the arrow. $\mathbf{b}$ Effect of light on the incorporation of [ ${ }^{32} \mathrm{Pi}$ ] into the $\mathrm{C} 1$ histidine kinase fusion protein. [Y- $\left.{ }^{32} \mathrm{P}\right]-$ ATP was used as the phosphoryl group donor. Reaction mixtures were incubated either in the presence of light (open symbols) or in the dark (filled circles). Error bars represent the standard deviation calculated from three independent experiments. For further detail: see Materials and methods

differences in activity, when assayed in light and dark, were only observed for the fusion protein $\mathrm{C} 1$ (while a very small difference was observed for C6; see Additional file 1: Table S1). The former, i.e. C1, in spite of its lower maximal activity, was therefore selected for further experiments. Significantly, for both hybrid kinases it turned out that illumination lowered their activity. In subsequently designed fusion proteins (e.g. C11) it turned out to be possible to observe significant light-stimulation of kinase activity (Additional file 1: Table S1). As for our subsequent in vivo experiments (see below) the lightinhibition of kinase activity was most valuable, these latter constructs have not been further characterized.

For the $\mathrm{C} 1$ light-modulatable histidine protein kinase we then characterized the kinetic basis of its light sensitivity. Time-course phosphorylation experiments in a time window of $120 \mathrm{~min}$ revealed that in most experiments the increase in the degree of phosphorylation of the kinase was approximately proportional with time during the first 30 min (Additional file 1: Fig. S1). Therefore, the dependency of the rate of autophosphorylation of the C1 kinase on the concentration of ATP was investigated with a range of nucleotide concentrations from 0 to $5 \mathrm{mM}$ (Fig. 2b). These experiments revealed that under both assay conditions (i.e. in light and in the dark) the half-maximal rate of phosphorylation is observed at about $0.5 \mathrm{mM}$ ATP, while the maximal rate of phosphorylation $\left(\mathrm{V}_{\max }\right)$ is lowered with more than $50 \%$ in the presence of saturating amounts of blue light (Fig. 2b). We do not refer to $\mathrm{Km}$ values here because under both conditions the rate of phosphorylation appears to be dependent on the nucleotide concentration in a slightly sigmoidal way, which may be due to allosteric regulation of the kinase activity. This latter point, however, was not further investigated.

Besides their autophosphorylation activity, several of the hybrid kinases were also tested for activity in an assay that measures phosphoryl transfer from the kinase/response-regulator domain of $\operatorname{Sln} 1$ to the phosphoryl-transfer domain of this phosphorelay system, i.e. Ypd1. All constructs except $C 9$ were active in this assay; (data not shown) however, because we did not 
have a rapid-quench system available (compare (JaniakSpens et al. 2005; Kaserer et al. 2010)), we could not time-resolve this process, and therefore not differentiate between the different hybrid kinases with respect to this activity.

\section{In vivo functionality of the hybrid kinase in the Skn7 signal transduction pathway}

As outlined in the Introduction, the Sln 1 phospho-relay system has two output pathways that target the HOG1 and the Skn7 nuclear transcriptional regulator, respectively. The Skn7 pathway is the most direct one of these two because Ypd1 directly phosphorylates Skn7 (Lu et al. 2003). We therefore first tested whether or not illumination, via the hybrid kinase $\mathrm{C} 1$, could elicit changes in the activity of Skn7. A suitable read-out of the latter is the level of expression of the mannosyl-transferase $\mathrm{OCH} 1$, via the reporter enzyme $\beta$-galactosidase, translationally fused to the former ( $\mathrm{Lu}$ et al. 2003; Li et al. 2002). The results summarized in Fig. 3 clearly show that this is indeed the case: Switching on saturating intensities of blue light decreases this expression level about twofold and a decrease is indeed expected as illumination decreases the rate of phosphorylation of the $\mathrm{C} 1$ kinase. It is of note that the replacement of the native $\operatorname{Sin} 1$ kinase by $\mathrm{C} 1$ does give a doubling of the level of $\mathrm{OCH} 1$ expression, but this is presumably due to the deregulated

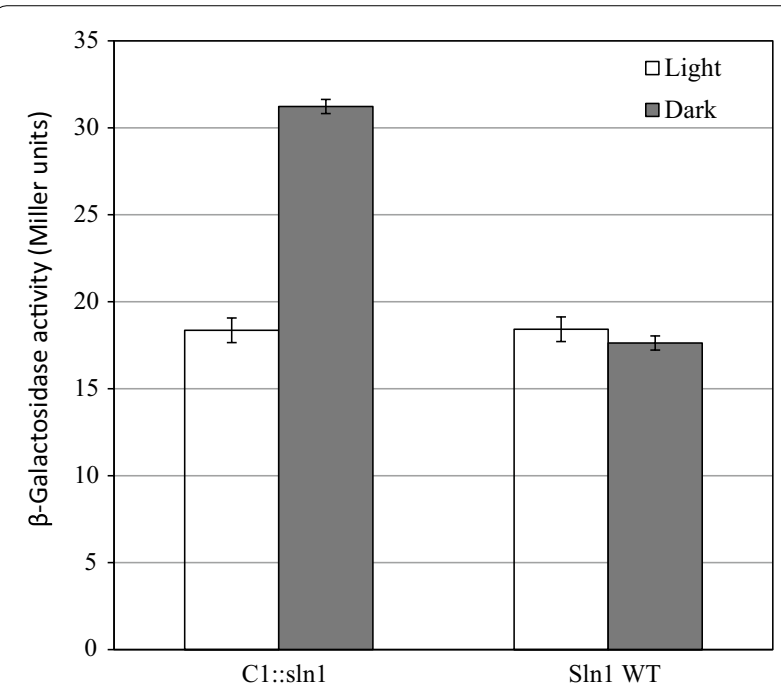

Fig. 3 Effect of illumination on the Skn7-dependent expression of the OCH1-lacZ transcriptional fusion (i.e. the mannosyl-transferase gene fused with the reading frame encoding $\beta$-galactosidase) in a $S$. cerevisiae strain with the wild-type $\ln 1$ gene, and in a strain with $\sin 1$ replaced by the gene encoding the $\mathrm{C} 1$-histidine kinase fusion protein. Cells were incubated either in the presence of light (white bars) or in the dark (grey bars). The level of expression of the OCH1-lacZ fusion is deduced from the specific $\beta$-galactosidase activity, expressed in Miller units. Error bars indicate standard deviations calculated from three independent biological experiments expression and/or activity of the truncated kinase domain. The additional control experiment of illuminating wild type cells, clearly shows that without the introduction of the fused LOV domain-containing protein in this assay S. cerevisiae does not respond to light (Fig. 3).

\section{In vivo functionality of the hybrid C1 kinase in the HOG1} pathway: observation of light-induced nuclear shuttling For this test we used two $S$. cerevisiae strains in which the endogenous Sln1 kinase and the HOG1 regulator protein had been genetically deleted and replaced by the $\mathrm{C} 1$ hybrid kinase and a translational HOG1::GFP fusion protein, respectively. The first one of these two strains had only the HOG1 gene replaced, and the second strain, both genes. With the resulting two strains stimulus/response experiments were carried out: With the first strain by eliciting an osmostress response, and in the second strain, which now is insensitive to osmostress, the occurrence of a light-response was tested (Figs. 4, 5). Through fluorescence microscopy of glutaraldehydefixed cells at emission and excitation wavelengths suitable for the analysis of their GFP- and DAPI content, respectively, we then analyzed the subcellular distribution of these two fluorophores, in which of course DAPI reveals the presence of the nuclear compartment, while GFP is present in both the nucleus and the cytosol. Figure 4a then shows the well-known response of the HOG1 protein in S. cerevisiae upon osmostress (Posas et al. 1996): An almost equal distribution of the HOG1::GFP fusion protein over the two compartments prior to the stress, followed by a rapid (i.e. within a few minutes) and significant accumulation in the nucleus after this stress. Panel b of Fig. 4 shows that the same response, i.e. HOG1 accumulation in the nucleus, can be elicited by exposing the yeast cells to saturating intensities of blue light of the yeast strain in which next to HOG1, also the $\operatorname{Sin} 1$ kinase has been eliminated and replaced by the $\mathrm{C} 1$ hybrid kinase. In Fig. 5a quantitative analysis of the dynamics of these two responses (i.e. to osmostress and to illumination) is presented. The osmostress response shows the typical transient response with maximally almost twofold accumulation in the nucleus of the HOG1::GFP fusion protein after around $5 \mathrm{~min}$, and a full relaxation of this concentration gradient at long timescales (e.g. $30 \mathrm{~min}$; compare ref (Posas et al. 1996)). The light-induced response in the strain carrying the hybrid $\mathrm{C} 1$ kinase, in contrast, shows the expected persistent response of a light-activatable system in continuous light, but appears to take more time to develop. More detailed analysis, at the level of the individual cells (Additional file 1: Fig. S2) shows that the nuclear accumulation of the fluorescent reporter (i.e. HOG1) in selected cells can increase up to fourfold (with salt stress) and slightly less (i.e. up to 

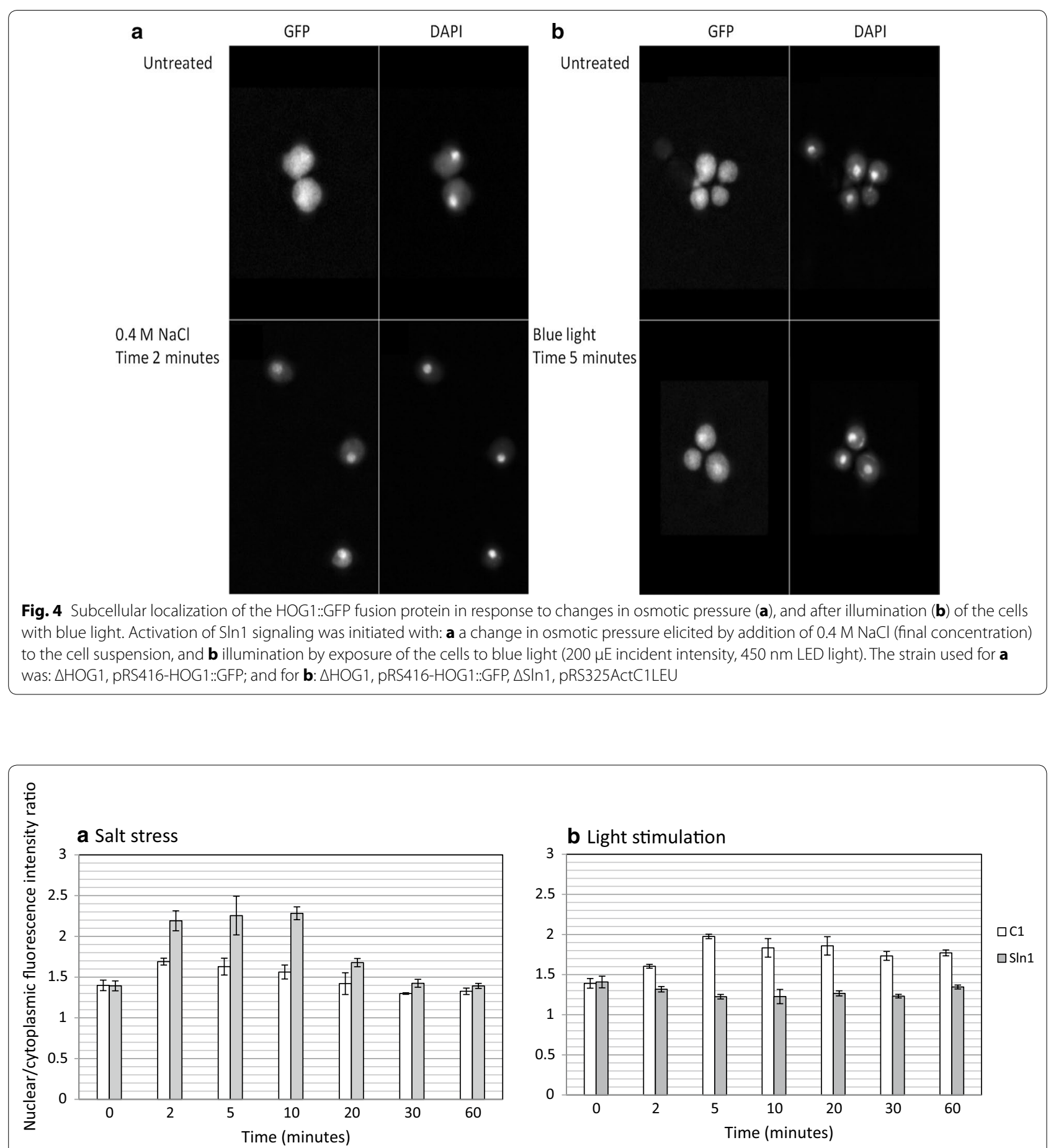

Fig. 5 Nucleo-cytoplasmic redistribution of the HOG1::GFP fusion protein after a salt stress (a) and after illumination of the cells (b). The salt stress (a) was applied by addition of $0.4 \mathrm{M} \mathrm{NaCl}$ (final concentration) to the cell suspension. In $\mathbf{b}$ the results are shown of exposure of the cell suspension to blue light (200 $\mu$ E incident intensity, $450 \mathrm{~nm}$ LED light). Samples were taken 0, 2, 5, 10, 20, 30 and 60 min after initiation of the experiment. White bars represent the yeast strain with the $\mathrm{C} 1$ histidine kinase fusion protein, and grey bars represent the yeast strain with the native SIn 1 histidine kinase. Error bars represent the standard deviation calculated from three independent experiments 
threefold) with illumination. Consistent with the results displayed in Fig. 5, also in Additional file 1: Fig. S2 we see the same slower kinetics with light activation. An additionally significant effect visible from this figure is the fact that the functionality of both signal transduction systems depends on the level of expression of the fluorescently labelled HOG1 protein: If the expression level of this protein is increased more than fivefold over minimum expression levels, neither the light- nor and stressinduced nuclear accumulation are detectable anymore.

\section{Discussion}

Design and activity of the LOV::SIn 1 histidine kinase fusion proteins

The light-stimulated fusion proteins described in this study were designed following work reported by the group of Moffat, Möglich and co-workers (Möglich et al. 2009; Möglich and Moffat 2007, 2010). Their approach is based on the identification of the boundaries of the independently folded domains in signal-transduction proteins like FixL, YtvA, etc., and of the helices linking them. These linker helices often form a coiled/coil tertiary structure in dimers of the corresponding signal transduction proteins. Coiled/coil structures, besides their $\alpha$-helical nature, display a seven- (hepta-) amino acid repeat structure, with a hydrophobic side chain at each 4th- and 7th-position (Diensthuber et al. 2013). This repeat structure then provides a rationale for domain swapping to make new functionalities via fusion proteins. A light stimulated histidine kinase can for instance be constructed by swapping sequences within these linker domains so that the stability of the independently folded domains would not be affected by the swap. Therefore, the coiled/coil linker helices, identified in YtvA and in $\operatorname{Sin} 1$, were aligned on the basis of the hepta-helical repeat motif that is identifiable in both of them (Fig. 2a) (Tao et al. 2002; Möglich et al. 2009). This alignment shows that the YtvA sequence, (directly) following the conserved DIT motif (amino acids \# 125-127, which are key to signal transduction within the YtvA protein (for review: see (van der Steen et al. 2012)) can be fused with a non-cognate kinase domain. The Sln 1 kinase domain qualifies for this in the region just upstream the phosphorylatable histidine of the kinase (i.e. amino acids \# 512-540).

Based on the above considerations we designed the $\mathrm{C} 1$ construct in which the upstream sequences, including the linker helix of $\operatorname{Sn} 1$ are replaced by the LOV domain plus Ja helix, from YtvA. Constructs C2, C5 and C6 instead have the LOV domain fused to the Sln1 kinase domain directly after the conserved DIT motive. They differ among each other in the length of the helical linker of the Sln1 domain (see Additional file 1: Table
S1) which will have an influence on the total length of the coiled/coil structure. In the C8 construct the part contributed by YtvA has been extended with 6 amino acids, as an attempt to enhance the difference in kinase activity between light and dark, following Möglich's design of the YF2 construct (Möglich et al. 2009).

As in the $\mathrm{C} 1$ construct light-inhibition of kinase activity was observed, we also tried to design constructs with light-stimulated kinase activity. Such light-stimulated kinase activity was e.g. reported for the YF constructs described in Möglich et al. (Möglich et al. 2009). Their YF1 construct is composed of the LOV domain from YtvA (\# 1-127) fused to the kinase domain of FixL (\# 258-505), i.e. it derives its helical linker from FixL. We therefore first composed the triple-fusion protein $\mathrm{C}$, consisting of the LOV domain of YtvA (\# 1-127), the linker helix of FixL (\# 259-281) and the histidine kinaseplus response regulator domain from $\operatorname{Sin} 1$ (\# 567-1221). Phosphorylation assays, however, showed that this construct did not display any measurable kinase activity (Additional file 1: Table S1). Two constructs were then designed, to conserve the sequence around the DIT motif of YtvA, and to expand it to the DITKQ motif. Accordingly, $\mathrm{C} 10$ was designed, and also $\mathrm{C} 11$, with the deletion of one amino acid downstream of the DITKQ motif (Fig. 2a). The TKQ motif was identified in the $\operatorname{Sln} 1$ histidine kinase too and therefore the YtvA sequence was linked to the kinase domain with optimal conservation of this domain and the coiled/coil structure. Of these two constructs, indeed $\mathrm{C} 11$ shows light-activation of kinase activity in the auto-phosphorylation assay (Additional file 1: Table S1). However, as the most important in vivo test is best carried out with a kinase of which the activity is decreased upon illumination, construct $\mathrm{C} 11$ was not used in the studies of the shuttling of HOG1; it may, however, be of interest for future studies of gene activation in S. cerevisiae (c.f. Fig. 3).

\section{The two signal-transduction pathways emerging from SIn 1: shuttling of Ypd1}

The introductory figure of this report (Fig. 1) does not make an explicit statement on the issue of whether or not also Ypd1 would act in signal transfer to nuclear components by active shuttling between the two compartments. The available experimental evidence suggests that it does not ( $\mathrm{Lu}$ et al. 2003), although alternative mechanisms for relay of the Ypd $\sim$ P signal to the nucleus have not been proposed (yet). In relation to this it is relevant to note that we too have tried to observe nuclear accumulation of Ypd1, fused to GFP, with fluorescence microscopy, and could not observe significant nuclear accumulation of this fusion protein either (compare to (Lu et al. 2003)), under conditions that the salt stress 
gave a very clear response for the HOG1 (GFP-fusion) protein.

\section{Signal transduction in the $\operatorname{SIn} 1$ system and the number of signal-transducing molecules}

Beyond the difference in light sensitivity-the main purpose of the experiment-of the two strains reported on in Fig. 3, it is clear that the one with the truncated $\operatorname{Sn} 1$ fusion protein shows considerably higher activity in the dark than the unperturbed wild type system. Two possible underlying differences can explain this latter aspect: (i) a higher intrinsic kinase activity of the LOV::Sln1 fusion construct than the authentic Sln1 kinase and (ii) a higher expression level of the fusion kinase. As the kinase is not expressed from its natural promoter, but from the ACT1 promoter, a rather strong, mostly constitutive (but glucose repressible) promotor (Wenzel et al. 1995; Planta et al. 1999) we think that the fusion kinase may be present at higher concentration than Sln1. Nevertheless, a higher intrinsic activity may also play a role as a similar activation has also been observed in some bacterial twocomponent kinases (Szurmant et al. 2008; Verhamme 2002).

Absolute numbers, and by inference concentrations, of the molecular components of a signal transduction chain are important, particularly in the two-component systems, e.g. because most kinases in the absence of their cognate signal, display considerable phosphatase activity. The approximately 10 - to 100 -fold molar excess of response regulator over kinase in most bacterial twocomponent systems testifies to this (e.g. (Yoshida et al. 2002; Wayne et al. 2010)). The results presented in Additional file 1: Fig. S2 show that both in the natural response system to osmotic stress and in the light response, mediated by the hybrid kinase, the HOG1/kinase molar ratio is of crucial importance too. If the concentration of the HOG1::GFP reporter protein is increased from its basal level (in cells that presumably contain only a single copy of the expression plasmid) to more than four to fivefold higher, the signal transduction system seems oversaturated with HOG1, and a response to both stimuli is no longer visible. The unperturbed $\operatorname{Sin} 1$ signal transduction system functions with 656 and 6780 molecules per cell of Sln1 kinase and the HOG1 transducer, respectively (Ghaemmaghami et al. 2003). This corresponds to $\sim 25 \mathrm{nM}$ and $0.5 \mu \mathrm{M}$, respectively in non-stimulated cells. Analysis of the average cellular concentration of the HOG1::GFP fusion protein with fluorescence-correlation microscopy (M. Hink et al., unpublished observation; for methodology see (Maeder et al. 2007)) suggests that its abundance-at the basal, pre-stimulus, level-is 0.15 $( \pm 0.06 ; \mathrm{n}=41) \mu \mathrm{M}$, i.e. slightly lower but still comparable to that of the HOG1 protein in the wild type, in spite of the differences in promoters used. These results suggest that the concentration of the HOG1 protein in the $\operatorname{Sin} 1$ signal transduction pathway is such that overexpression of HOG1 above physiological levels will make the Sln1 signal transduction pathway non-functional.

The results shown in Fig. 5 and Additional file 1: Fig. S2 suggest that the on-dynamics of the light response is slower than that of the osmostress response. If so, this may have several causes, like a lower degree of kinase modulation by light, or a suboptimal expression ratio of the proteins composing the light-responsive signal transduction pathway. Furthermore, the open bars in Fig. 5, panel a, do seem to show a very slight remaining stimulation of HOG1 accumulation in the nucleus upon stressing the strain that expresses the light-sensitive, truncated variant of $\operatorname{Sln} 1$. This can be explained by weak spill-over of signals from the osmostress-responsive Sho1 system of S. cerevisiae into the $\operatorname{Sln} 1$ system at the level of the Ssk1 MAP kinase pathway (Hao et al. 2007).

The hybrid kinase described in this study is an excellent candidate for future studies on quantitation of the consequences of e.g. localized kinase activation in the cytoplasm, for the dynamics and amplitude of the overall cellular response. This will allow further fine-tuning, e.g. with respect to the role of (anomalous) cytoplasmic diffusion, of systems biology models developed to describe the osmo/stress response in S. cerevisiae (Uschner and Klipp 2014; Dexter et al. 2015). Various super-resolution microscopy techniques are available to facilitate such experiments (e.g. Small and Parthasarathy 2014)). Also the use of specific subcellular localization tags and/or interaction domains can be exploited for this (Schierling and Pingoud 2012).

\section{Additional file}

Additional file 1. Additional tables and figures

Abbreviations

GFP: green fluorescent protein; LOV: light oxygen voltage; LED: light emitting diodes.

Authors' contributions

All authors read and approved the final manuscript.

\section{Acknowledgements}

The authors want to thank J. Arents for production and purification of the hybrid kinases, G. Smits for expert advice about yeast genetics, A. Möglich for his advice about design of light-dependent kinases, A. West for the generous hospitality in her lab and for sharing knowledge about the phosphorylation assays, and colleagues from the Molecular Cytology Group of the Swammerdam Institute (D. Gadella, M. Hink and E. Manders) for expert help with and advice and discussion on the topic of fluorescence microscopy.

Competing interests

Both authors declare that they have no competing interests. 


\section{Availability of data and materials}

Not applicable.

\section{Consent for publication}

Not applicable.

\section{Ethics approval and consent to participate}

This article does not contain any studies with human participants or animals performed by any of the authors.

\section{Funding}

This work was supported by the Foundation for Fundamental Research on Matter (FOM) through programme number 110 (Spatial design of biochemical regulation networks (SPAT), awarded to Prof. dr. M. Dogterom (AMOLF, Amsterdam, The Netherlands).

\section{Publisher's Note}

Springer Nature remains neutral with regard to jurisdictional claims in published maps and institutional affiliations.

Received: 20 March 2018 Accepted: 25 March 2018

Published online: 02 April 2018

\section{References}

Abe K, Miyake K, Nakamura M, Kojima K, Ferri S, Ikebukuro K, Sode K (2014) Engineering of a green-light inducible gene expression system in Synechocystis sp. PCC6803. Microb Biotechnol 7(2):177-183

Avila-Perez M, Hellingwerf KJ, Kort R (2006) Blue light activates the sigmaBdependent stress response of Bacillus subtilis via YtvA. J Bacteriol 188(17):6411-6414

Bacchus W, Fussenegger M (2012) The use of light for engineered control and reprogramming of cellular functions. Curr Opin Biotechnol 23(5):695-702

Bradford MM (1976) Rapid and sensitive method for the quantitation of microgram quantities of protein utilizing the principle of protein-dye binding. Anal Biochem 72(1-2):248-254

Burbulys D, Trach KA, Hoch JA (1991) Initiation of sporulation in B. subtilis is controlled by a multicomponent phosphorelay. Cell 64(3):545-552

Chee MK, Haase SB (2012) New and redesigned pRS plasmid shuttle vectors for genetic manipulation of Saccharomyces cerevisiae. G3 Genes Genomes Genet. 2(5):515-526

Dexter JP, Xu P, Gunawardena J, Mcclean MN (2015) Robust network structure of the SIn1-Ypd1-Ssk1 three-component phospho-relay prevents unintended activation of the HOG MAPK pathway in Saccharomyces. BMC Syst Biol 9:17

Diensthuber RP, Bommer M, Gleichmann T, Möglich A (2013) Full-length structure of a sensor histidine kinase pinpoints coaxial coiled coils as signal transducers and modulators. Structure 21(7):1127-1136

Ernst OP, Sanchez Murcia PA, Daldrop P, Tsunoda SP, Kateriya S, Hegemann P (2008) Photoactivation of channelrhodopsin. J Biol Chem 283(3):1637-1643

Fassler JS, West AH (2010) Genetic and biochemical analysis of the $\sin 1$ pathway in Saccharomyces cerevisiae. Methods Enzymol 471:291-317

Ferrigno P, Posas F, Koepp D, Saito H, Silver PA (1998) Regulated nucleo/cytoplasmic exchange of HOG1 MAPK requires the importin beta homologs NMD5 and XPO1. EMBO J 17(19):5606-5614

Gao R, Stock AM (2009) Biological insights from structures of two-component proteins. Rev Microbiol 63:133-154

Ghaemmaghami S, Huh WK, Bower K, Howson RW, Belle A, Dephoure N, O'shea EK, Weissman JS (2003) Global analysis of protein expression in yeast. Nature 425(6959):737-741

Hao N, Behar M, Parnell SC, Torres MP, Borchers CH, Elston TC, Dohlman HG (2007) A systems-biology analysis of feedback inhibition in the Sho osmotic-stress-response pathway. Curr Biol 17(8):659-667

Hoff WD, Jung KH, Spudich JL (1997) Molecular mechanism of photosignaling by archaeal sensory rhodopsins. Rev Biophys Biomol Struct 26:223-258

Janiak-Spens F, Cook PF, West AH (2005) Kinetic analysis of YPD1-dependent phosphotransfer reactions in the yeast osmoregulatory phosphorelay system. Biochemistry 44(1):377-386
Kaserer AO, Andi B, Cook PF, West AH (2010) Kinetic studies of the yeast HisAsp phosphorelay signaling pathway. Methods Enzymol 471:59-75

Kim CK, Adhikari A, Deisseroth K (2017) Integration of optogenetics with complementary methodologies in systems neuroscience. Nat Rev Neurosci 18(4):222-235

Koziol J (1971) Fluorometric analyses of riboflavin and its coenzymes. Methods Enzymol 18:253-285

Levskaya A, Chevalier AA, Tabor JJ, Simpson ZB, Lavery LA, Levy M, Davidson EA, Scouras A, Ellington AD, Marcotte EM, Voigt CA (2005) Synthetic biology: engineering Escherichia coli to see light. Nature 438(7067):441-442

Li S, Dean S, Li Z, Horecka J, Deschenes RJ, Fassler JS (2002) The eukaryotic two-component histidine kinase $\operatorname{SIn} 1 \mathrm{p}$ regulates $\mathrm{OCH} 1$ via the transcription factor, Skn7p. Mol Biol Cell 13(2):412-424

Lu JMY, Deschenes RJ, Fassler JS (2003) Saccharomyces cerevisiae histidine phosphotransferase Ypd1p shuttles between the nucleus and cytoplasm for SLN1-dependent phosphorylation of Ssk1p and Skn7p. Eukaryot Cell 2(6):1304-1314

Maeder Cl, Hink MA, Kinkhabwala A, Mayr R, Bastiaens PI, Knop M (2007) Spatial regulation of Fus3 MAP kinase activity through a reaction-diffusion mechanism in yeast pheromone signalling. Nat Cell Biol 9(11):1319-1326

Miller JH (1972) Experiments in Molecular Genetics. Cold Spring Harbour Laboratory Press, Cold Spring Harbor

Miller G (2006) Optogenetics. Shining new light on neural circuits. Science 314(5806):1674-1676

Miyake K, Abe K, Ferri S, Nakajima M, Nakamura M, Yoshida W, Kojima K, Ikebukuro K, Sode K (2014) A green-light inducible lytic system for cyanobacterial cells. Microb Biotechnol 7:56. https://doi. org/10.1186/1754-6834-7-56

Möglich A, Moffat K (2007) Structural basis for light-dependent signaling in the dimeric LOV domain of the photosensor YtvA. J Mol Biol 373(1):112-126

Möglich A, Moffat K (2010) Engineered photoreceptors as novel optogenetic tools. Photochem Photobiol Sci 9(10):1286-1300

Möglich A, Ayers RA, Moffat K (2009) Design and signaling mechanism of lightregulated histidine kinases. J Mol Biol 385(5):1433-1444

Nixon BT, Ronson CW, Ausubel FM (1986) Two-component regulatory systems responsive to environmental stimuli share strongly conserved domains with the nitrogen assimilation regulatory genes ntrB and ntrC. Natl Acad Sci 83(20):7850-7854

Planta RJ, Brown AJ, Cadahia JL, Cerdan ME, De Jonge M, Gent ME, Hayes A, Kolen CP, Lombardia LJ, Sefton M, Oliver SG, Thevelein J, Tournu H, Van Delft YJ, Verbart DJ, Winderickx J (1999) Transcript analysis of 250 novel yeast genes from chromosome XIV. Yeast 15(4):329-350

Posas F, Wurgler-Murphy S, Maeda T, Witten E, Thai T, Saito H (1996) Yeast HOG1 MAP kinase cascade ss regulated by a multistep phosphorelay mechanism in the SLN1-YPD1-SSK1 "two-component" osmosensor. Cell 86(6):865-875

Ridge KD, Abdulaev NG, Sousa M, Palczewski K (2003) Phototransduction: crystal clear. Trends Biochem Sci 28(9):479-487

Savakis P, Hellingwerf KJ (2015) Engineering cyanobacteria for direct biofuel production from CO2. Curr Opin Biotechnol 33:8-14

Schierling B, Pingoud A (2012) Controlling the DNA cleavage activity of light-inducible chimeric endonucleases by bidirectional photoactivation. Bioconjug Chem 23(6):1105-1109

Schindelin J, Arganda-Carreras I, Frise F, Kaynig V, Longair M, Pietzsch T, Preibisch S, Rueden C, Saalfeld S, Schmid B, Tinevez J, White DJ, Hartenstein V, Eliceiri K, Tomancak P, Cardona A (2012) Fiji: an Open Source platform for biological image analysis. Nat Methods 9:676-682

Small AR, Parthasarathy R (2014) Superresolution localization methods. Annu Rev Phys Chem 65:107-125

Szurmant H, Bu L, Brooks CL, Hoch JA (2008) An essential sensor histidine kinase controlled by transmembrane helix interactions with its auxiliary proteins. Proc Natl Acad Sci 105(15):5891-5896

Tao W, Malone CL, Ault AD, Deschenes RJ, Fassler JS (2002) A cytoplasmic coiled-coil domain is required for histidine kinase activity of the yeast osmosensor, SLN1. Mol Microbiol 43(2):459-473

Uschner F, Klipp E (2014) Information processing in the adaptation of Saccharomyces cerevisiae to osmotic stress: an analysis of the phosphorelay system. Syst Synth Biol 8(4):297-306

Van Der Horst MA, Hellingwerf KJ (2004) Photoreceptor proteins, "star actors of modern times": a review of the functional dynamics in the structure 
of representative members of six different photoreceptor families. Acc Chem Res 37(1):13-20

Van Der Steen JB, Avila-Perez M, Knippert D, Vreugdenhil A, Van Alphen P, Hellingwerf KJ (2012) Differentiation of function among the RsbR paralogs in the general stress response of Bacillus subtilis with regard to light perception. J Bacteriol 194(7):1708-1716

Verhamme D (2002) Signal transfer through the Uhp regulatory system in the signal transduction network of Escherichia coli. Dissertation, University of Amsterdam

Wayne KJ, Sham LT, Tsui HC, Gutu AD, Barendt SM, Keen SK, Winkler ME (2010) Localization and cellular amounts of the WalRKJ (VicRKX) two-component regulatory system proteins in serotype 2 Streptococcus pneumoniae. J Bacteriol 192(17):4388-4394
Wenzel TJ, Teunissen AW, De Steensma HY (1995) PDA1 mRNA: a standard for quantitation of mRNA in Saccharomyces cerevisiae superior to ACT1 mRNA. Nucleic Acids Res 23(5):883-884

Wu YI, Frey D, Lungu Ol, Jaehrig A, Schlichting I, Kuhlman B, Hahn KM (2009) A genetically encoded photoactivatable Rac controls the motility of living cells. Nature 461(7260):104-108

Yoshida T, Cai S, Inouye M (2002) Interaction of EnvZ, a sensory histidine kinase, with phosphorylated $\mathrm{OmpR}$, the cognate response regulator. Mol Microbiol 46(5):1283-1294

Zhang F, Gradinaru V, Adamantidis AR, Durand R, Airan RD, De Lecea L, Deisseroth K (2010) Optogenetic interrogation of neural circuits: technology for probing mammalian brain structures. Nat Protoc 5(3):439-456

\section{Submit your manuscript to a SpringerOpen ${ }^{\circ}$ journal and benefit from:}

- Convenient online submission

- Rigorous peer review

- Open access: articles freely available online

- High visibility within the field

- Retaining the copyright to your article

Submit your next manuscript at $\gg$ springeropen.com 